\title{
The Shape of Strongly Disturbed Dayside Magnetopause
}

\author{
Alexei V. Dmitriev ${ }^{1, *}$ and Alla V. Suvorova ${ }^{2}$ \\ ${ }^{1}$ Institute of Space Science, National Central University, Jhongli, Taiwan \\ ${ }^{2}$ Center for Space and Remote Sensing Research, National Central University, Jhongli, Taiwan
}

Received 5 April 2012, accepted 26 September 2012

\begin{abstract}
During strong geomagnetic disturbances, the Earth's magnetosphere exhibits unusual and nonlinear interaction with the incident flow of magnetized solar wind plasma. Global Magneto-hydro-dynamic (MHD) modeling of the magnetosphere predicts that the storm-time effects at the magnetopause result from the abnormal plasma transport and/or extremely strong field aligned currents. In-situ observations of the magnetospheric boundary, magnetopause, by Geosynchronous Operational Environmental Satellite (GOES) allowed us to find experimentally such effects as a saturation of the dayside reconnection, unusual bluntness and prominent duskward skewing of the nose magnetopause. The saturation and duskward skewing were attributed to the storm-time magnetopause formation under strong southward interplanetary magnetic field (IMF). The unusual bluntness was observed during both high solar wind pressure and strong southward IMF. We suggest that these phenomena are caused by a substantial contribution of the cross-tail current magnetic field and the hot magnetospheric plasma from the asymmetrical ring current into the pressure balance at the dayside magnetopause.
\end{abstract}

Key words: Magnetopause, Magnetospheric currents, Geomagnetic storm

Citation: Dmitriev, A. V. and A. V. Suvorova, 2013: The shape of strongly disturbed dayside magnetopause. Terr. Atmos. Ocean. Sci., 24, 225-232, doi: 10.3319/TAO.2012.09.26.02(SEC)

\section{INTRODUCTION}

The magnetopause shape is usually represented by a surface of revolution around the aberrated Sun-Earth line, or $\mathrm{X}-\mathrm{GSM}$ axis. A functional form of the shape and its dependence on solar wind driving parameters vary from one model to another (e.g., Shue et al. 2000). Key driving parameters of the magnetopause are solar wind dynamic pressure $\left(P_{d}\right)$ and $B_{z}$ component of interplanetary magnetic field (IMF). Different parts of the magnetopause are driven by the solar wind parameters in different manners. Here we focus on the dayside magnetopause.

For quiet and moderately disturbed conditions, practically all the empirical models demonstrate similar accuracy in prediction of the dayside magnetopause (Šafránková et al. 2002). During strong geomagnetic disturbances, the magnetopause exhibits unusual and nonlinear behavior such as reconnection saturation and prominent dawn-dusk asymmetry (e.g., Dmitriev et al. 2004, 2011; Suvorova et al. 2005).

\footnotetext{
* Corresponding author

E-mail:dalex@jupiter.ss.ncu.edu.tw
}

It is widely accepted that the subsolar magnetopause moves earthward due to the reconnection of geomagnetic field with a southward component (negative $B_{z}$ ) of the IMF. Stronger southward IMF results in a smaller distance to the subsolar magnetopause. However, when the magnitude of negative $B_{z}$ exceeds a certain threshold, the magnetopause distance no longer decreases, i.e., the southward IMF influence (reconnection) is saturated (e.g., Yang et al. 2003; Suvorova et al. 2005). The threshold for saturation is estimated to be about $-20 \mathrm{nT}$ (i.e., $E \sim 10 \mathrm{mV} \mathrm{m}^{-1}$ ) and its magnitude increases with solar wind pressure.

The dawn-dusk asymmetry in the shape of dayside magnetopause has been convincingly shown on the basis of geosynchronous magnetopause crossings (GMCs) (Dmitriev et al. 2004). They found that the GMCs occur most often in a prenoon sector. Moreover, higher (lower) solar wind pressure is required to push the magnetopause inside the geosynchronous orbit in postnoon (prenoon) and dusk (dawn) sectors. As a result, during magnetic storms the magnetopause is located closer to the Earth in the dawn and prenoon sectors and is more distant in the postnoon and 
dusk sectors. This duskward skewing can be represented by a translational shifting of the magnetopause toward dusk such that the focus of the magnetopause conic section would be shifted from the Sun-Earth line towards dusk by several tenths of the Earth's radii (Re) (Dmitriev et al. 2005a).

For slightly and strongly disturbed conditions, the dayside magnetopause is more accurately predicted by those models which incorporate non-linear effects (Lin et al. 2010). A well-regarded model developed by Kuznetsov and Suvorova (1998) reproduces both the reconnection saturation and duskward magnetopause skewing. The highest accuracy is demonstrated by a new 3-D magnetopause model by Lin et al. (2010), which incorporates the effects of reconnection saturation and a tilt of the geomagnetic field. Nevertheless, the non-linear effects at the storm-time magnetopause require still further investigation because of the limited amount of experimental data.

The reconnection saturation is studied on the basis of global MHD simulations. Siscoe et al. (2004) considered magnetic effect of the region 1 field-aligned current (FAC) as a possible mechanism responsible for saturations. At the stagnation point (subsolar magnetopause) the region $1 \mathrm{FAC}$ results in a depletion of the geomagnetic field as well as substantial changing of the magnetopause shape such that a dimple develops at the stagnation point and the magnetopause becomes blunt and the bow shock recedes. Dimples tend to fill with stagnated plasma, which puts a buffer between the boundary and the flow, that leads to decrease and limitation of the reconnection at the dayside magnetopause. Analyzing geomagnetic field observations during the strong southward IMF, Ober et al. (2006) have found experimental evidence of that mechanism. They observe a strong dominance of the region $1 \mathrm{FAC}$ generated magnetic fields both at high and low latitudes and diminishing of the ChapmanFerraro current system. Two sets of spacecraft observations at high and low latitudes suggest that the dayside magnetopause assumes a very blunt shape consistent with the predictions of the MHD simulations.

Modeling the dayside magnetopause with Artificial Neural Networks, Dmitriev and Suvorova (2000) found a "dimple" arising in the subsolar region when IMF $B_{z}<$ $-10 \mathrm{nT}$. Rufenach et al. (1989) interpreted the feature as a result of the magnetopause erosion. The dimple is a largescale local structure. It is unlikely that such structure is formed by the cross-tail and/or field-aligned currents, which produce a global magnetic effect in the dayside hemisphere. Hence, it should be another phenomenon responsible for that distortion.

Borovsky et al. (2008) used high-resolution 3-D MHD simulations and found that the reconnection can be saturated by a "plasmaspheric effect;" high-density magnetospheric plasma flows from plasmasphere into the magnetopause reconnection site and mass loads the reconnection such that the reconnection rate is reduced locally. Based on statistical analysis of magnetopause crossings of geosynchronous satellites, Suvorova et al. (2003) proposed that the reconnection saturation is caused by an enhanced thermal pressure of the magnetospheric plasma and ring current particles during strong magnetic storms.

Saturation effects are taken into account in the last versions of Tsyganenko's geomagnetic field model adopted for strong magnetic storms (e.g., Tsyganenko and Sitnov 2005). In the model all the internal magnetospheric currents (crosstail current, ring current and region 1 and 2 FACs) are saturated at different thresholds, which have been derived from the fitting of experimental data. It is important to note that in the Tsyganenko model, the magnetopause is a predefined surface fitted to the empirical boundary of Shue et al. (1998) but, in contrast to the original model, it is controlled only by the solar wind ram pressure. Hence, the Tsyganenko model can be used only for comparison with geomagnetic field observations and it is useless in studies of the magnetopause shape and location.

Despite being well-known for many years, the effect of dawn-dusk asymmetry is still poorly understood. The storm-time dawn-dusk asymmetry of the geomagnetic field is represented by the Tsyganenko model in the form of an asymmetrical ring current (Tsyganenko et al. 2003; Tsyganenko and Sitnov 2005).The contribution from the ring current was split into two parts, corresponding to its axially symmetric component and the partial ring current field. The partial ring current includes a westward azimuthal current at low latitudes, closed via field-aligned currents and localized in the evening-premidnight sector. This component is quite important in the modeling of the storm-time magnetosphere since it provides the observed strong dawn-dusk asymmetry of the geomagnetic field, extending over a wide range of altitudes up to geosynchronous orbit. However, the Tsyganenko model does not predict any changes of the magnetopause size and shape.

Here we report magnetopause shape distortions observed by GOES fleet of geosynchronous satellites. The GOES experimental data are presented in section 2. Distortions of the magnetopause shape are studied in section 3 . Section 4 offers a discussion and section 5 contains concluding remarks.

\section{GOES MEASUREMENTS OF THE MAGNETO- PAUSE}

In the study we use magnetic field data of 1-min time resolution acquired from GOES 8, 9, and 10 geosynchronous satellites in 1994 - 2001. The method of magnetopause identification is described in detail by Suvorova et al. (2005). A magnetopause crossing was identified when one of two requirements was satisfied: (1) the GOES magnetic field deviated significantly from the geomagnetic field with a dominant northward component in the dayside mag- 
netosphere and (2) the $B_{y}$ and $B_{z}$ components measured by the GOES correlated with the corresponding IMF components measured by an upstream monitor. High-resolution ( 1-min) experimental data about upstream solar wind conditions were acquired from Geotail, Wind, and Advanced Composition Explorer (ACE) upstream monitors. The solar wind propagation time from an upstream monitor to the Earth was taken into account.

The magnetopause has a systematic distortion related to the Earth's rotation around the Sun with an orbital speed of $\sim 30 \mathrm{~km} \mathrm{~s}^{-1}$. In order to eliminate the aberration effect, all the coordinates and vector parameters were converted in the aberrated GSM (aGSM) coordinate system by taking into account both the orbital velocity of the Earth and non-radial solar wind propagation (Dmitriev et al. 2003).

Figure 1 demonstrates that upstream solar wind conditions during GMCs vary over a very wide range of total solar wind pressures $\left(P_{s w}\right)$ from $\sim 5$ to $\sim 100 \mathrm{nPa}$ and $B_{z}$ from -35 to $30 \mathrm{nT}$. The $P_{s w}$ is calculated as the sum of the solar wind dynamic (including the helium contribution), thermal and magnetic pressures. Note that strong geomagnetic disturbances are produced by magnetic clouds which contain very hot plasma and strong magnetic field. Hence, the contribution of the thermal and magnetic pressures to the $P_{s w}$ can reach up to $30 \%$.

In Fig. 1 one can see that the solar wind conditions required for GMCs are restricted rather sharply by a lower envelope boundary below which GMCs are not observed. The envelope boundary corresponds to minimal solar wind conditions which are necessary for GMCs. It was derived

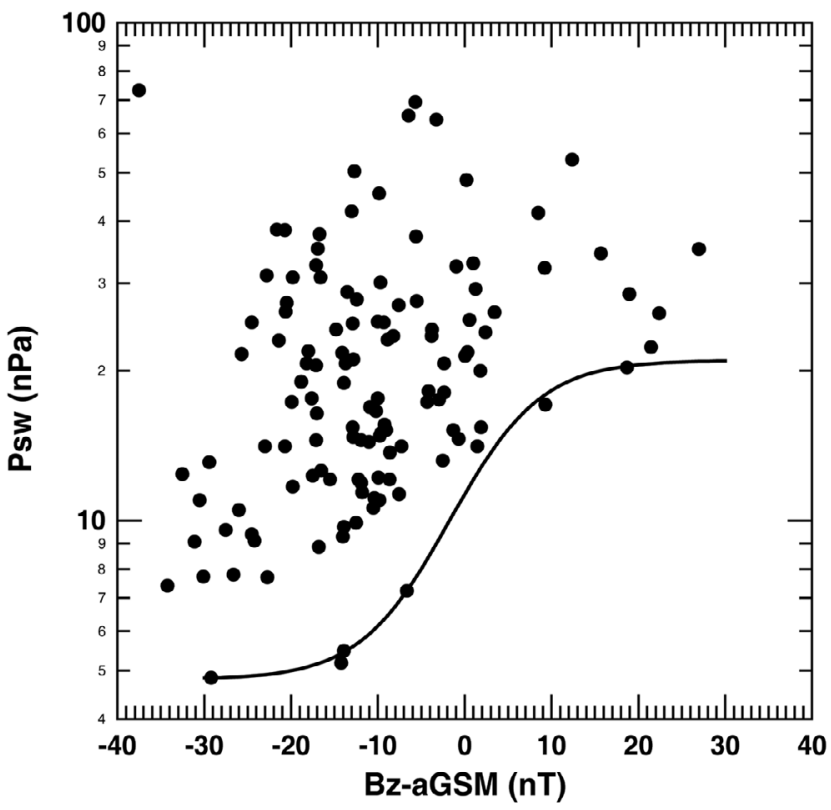

Fig. 1. Total solar wind pressure $\left(P_{s w}\right)$ and IMF $B_{z}$ in aGSM coordinates during GMCs. The thick solid curve indicates an envelope boundary derived by Suvorova et al. (2005). by Suvorova et al. (2005) on the basis of 23 magnetopause crossings observed by 7 geosynchronous satellites during 18 storm events and using solar wind data provided by 3 different upstream monitors. Thus, four outliers observed at low solar wind pressures and $B_{z}<0$ are actually just a portion of quite large statistics. In order to minimize instrumental effects such as aging and radiation effects of the ACE/SWEPAM plasma instrument (e.g., Dmitriev et al. 2005b, c), we restrict the data to the year 2002, at which time the degradation of the ACE plasma instrument was not yet significant.

Numerically the boundary can be represented by the following expression (Suvorova et al. 2005):

$P_{s w}=21-\frac{16.2}{1+\exp \left[0.2\left(B_{z}-2\right)\right]}$

The right horizontal branch of the envelope boundary, asymptotically approaching $P_{s w}=21 \mathrm{nPa}$, corresponds to a regime of pressure balance for the magnetopause under strong northward IMF. The left branch approaches $P_{s w}$ $\sim 4.8 \mathrm{nPa}$ under very strong negative $B_{z}$ and is associated with the regime of reconnection saturation.

A scatter plot of the collected GMCs in aberrated GSM coordinates is shown in Fig. 2. The crossings occupy a wide latitudinal sector from $-30^{\circ}$ to $30^{\circ}$ on the dayside. The wide latitudinal spread is explained by a superposition of two effects: $23,4^{\circ}$ tilt of the Earth's rotation axis to the ecliptic plane and $\sim 11^{\circ}$ tilt of the geomagnetic dipole relative to the

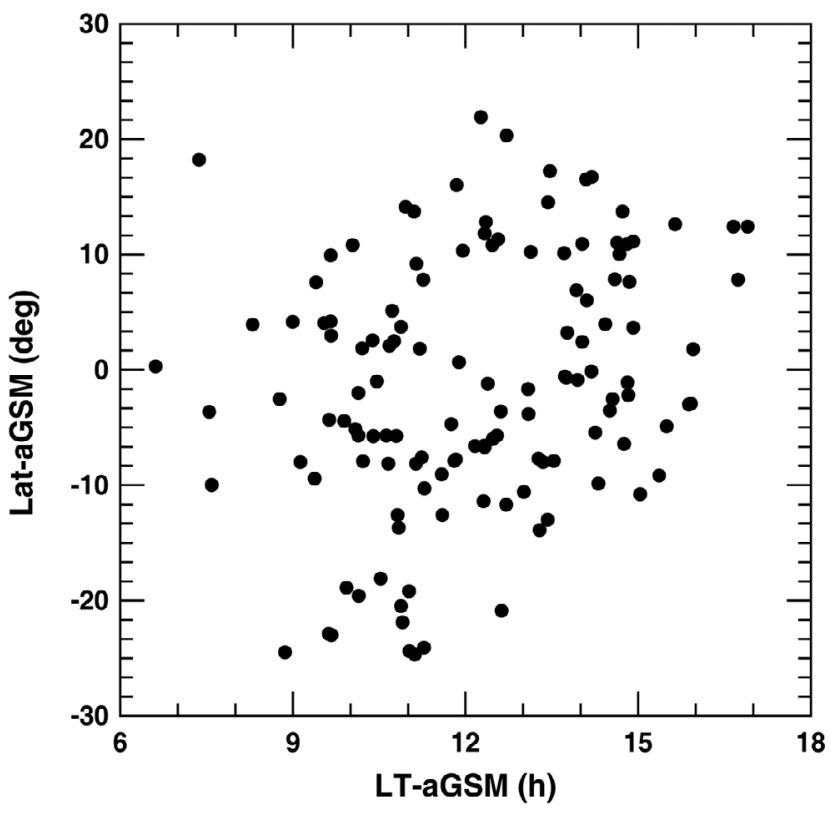

Fig. 2. Scatter plot of the collected GOES magnetopause crossings (GMCs) in aberrated GSM (aGSM) coordinates latitude versus local time. The GMCs occupy a wide latitudinal sector of $\pm 30^{\circ}$. 
rotation axis. The wide angular spread of GMCs makes it possible to conduct statistical studies of the shape of dayside magnetopause.

\section{DAYSIDE MAGNETOPAUSE DISTORTIONS}

The orientation of the magnetopause is determined from the rotation of the magnetic field across the magnetopause. Note that the magnetopause is usually considered as a tangential discontinuity (TD) wherein both the magnetic field and plasma parameters change abruptly. During magnetic reconnection with strong southward IMF, the magnetopause should be considered as a rotational discontinuity (RD) because of the nonzero magnetic field component normal to the magnetopause (e.g., Sonnerup and Ledley 1974).

A normal to the plane of magnetic field rotation across either TD or RD is determined from a minimal variance analysis (MVA). Note that the results of MVA vary by the length of the time interval chosen for the analysis. If the results vary greatly for different time intervals, they are unreliable. By varying the boundaries of the time intervals for each GMC, we found time ranges for which the results of MVA changed slightly and gradually (i.e., the solution of MVA was stable). Using this method, we calculate a normal to the magnetopause, i.e., local orientation of the magnetopause.

Figure 3 shows orientations of the normal to the magnetopause relative to the $\mathrm{X}$ axis in aGSM coordinates. The normal is characterized by a wide random scatter. The scatter with a relatively small tilt can be explained by tilted interplanetary fronts of enhanced solar wind pressure. Significant inclinations of the normal are revealed in $\sim 15^{\circ}$ vicinity of the nose point (longitude $=0$ and latitude $=0$ ). The prominent magnetopause distortions can result from time- dependent reconnection and multiple flux transfer events (FTEs) formed during strong southward IMF (Omidi et al. 2009).

Despite the scattering in Fig. 3, one can easily recognize a regular pattern of the normal orientation in relation to the curvature of the dayside magnetopause. In the zonal (X$\mathrm{Y})$ plane, the normal is mainly tilted duskward (dawnward) in the postnoon (prenoon) sector. In the meridional (X-Z) plane, the normal has a mainly northward (southward) tilt in the northern (southern) hemisphere. On average, the tilt increases with the angle from the nose point.

Figure 4 shows orientations of the normal to the magnetopause, which is mainly compressed by strong solar wind pressure $P_{s w}>16 \mathrm{nPa}$. Note that for the present case, the effect of reconnection is minor $\left(B_{z}>-5 \mathrm{nT}\right)$. Again, we find a regular pattern for the magnetopause normal, which is characterized by the tilt increasing with the latitude and longitude. On average, the tilt is almost symmetrical relative to the nose point. Hence, the magnetopause has a smooth shape symmetrical relative to the $\mathrm{X}$ axis. It is interesting to note an irregular orientation of the normal in the region restricted by $\sim 15^{\circ}$ of the longitude and $\sim 10^{\circ}$ of the latitude. It seems, such behavior indicates an unusually blunt magnetopause in the nose region under strong compression.

Another pattern of the magnetopause shape is revealed in Fig. 5 for the strong southward IMF $\left(B_{z}<-5 \mathrm{nT}\right)$ and moderate solar wind pressures $\left(P_{s w}<16 \mathrm{nPa}\right)$. In the zonal plane, the normal is mainly tilted dawnward in the prenoon sector, while in the postnoon sector, the normal is not regularly tilted duskward. In contrast, 11 out of 16 normals demonstrate dawnward or an unusually small duskward tilts. Note that the normals with unusual orientation are distributed widely from about $-10^{\circ}$ to $10^{\circ}$ in latitude. Statistically, the observed dawn-dusk asymmetry can be represented as an extended bluntness at the postnoon magnetopause. Per-

(a)

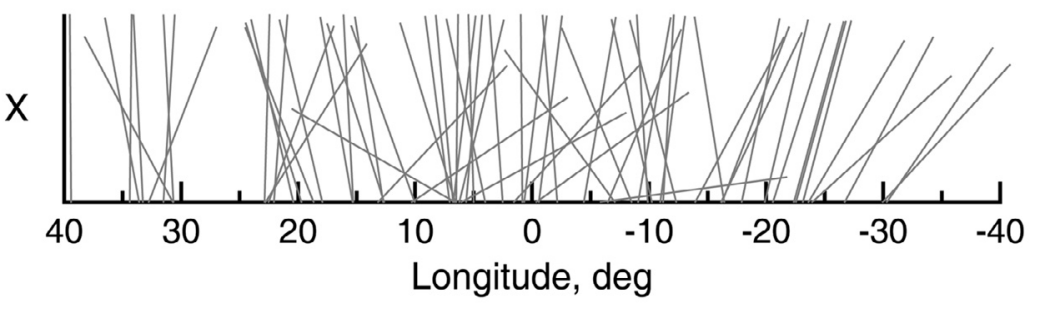

(b)

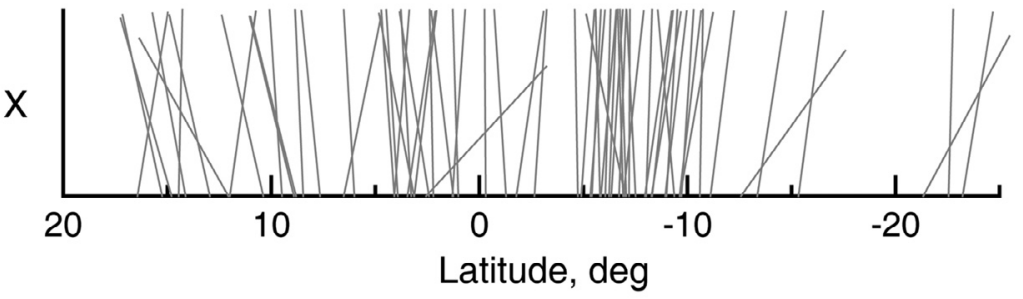

Fig. 3. Orientations of the normal to the magnetopause relative to the aGSM X-axis in the zonal plane (a) and meridional plane (b) for all selected GMCs. 
(a)

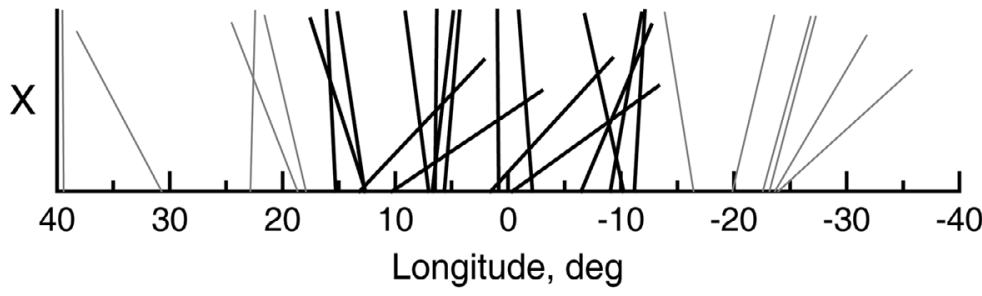

(b)

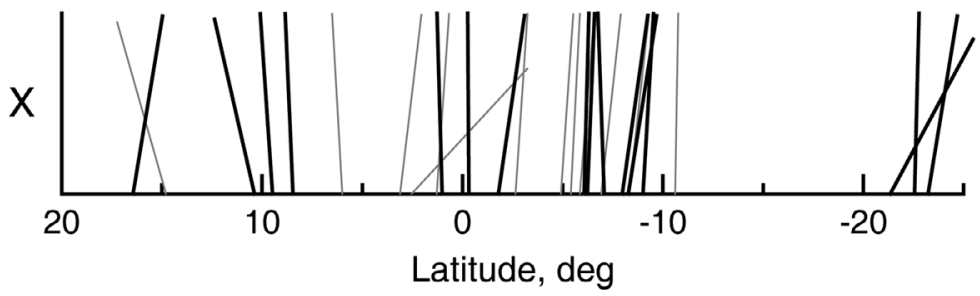

Fig. 4. The same as in Fig. 3 but for strong magnetopause compression: $P_{s w}>16 \mathrm{nPa}$ and $B_{z}>-5 \mathrm{nT}$. The normals in longitudinal range from $-15^{\circ}$ to $15^{\circ}$ are in bold. On average, the magnetopause shape is smooth and symmetrical around the $\mathrm{X}$-axis. The subsolar magnetopause seems unusually blunt.

(a)

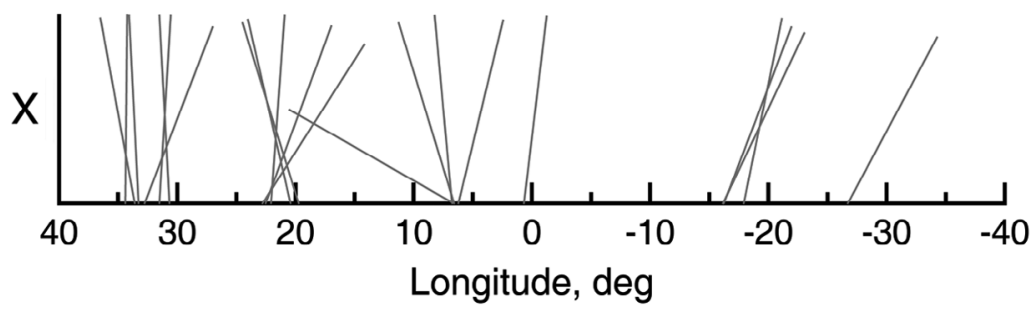

(b)

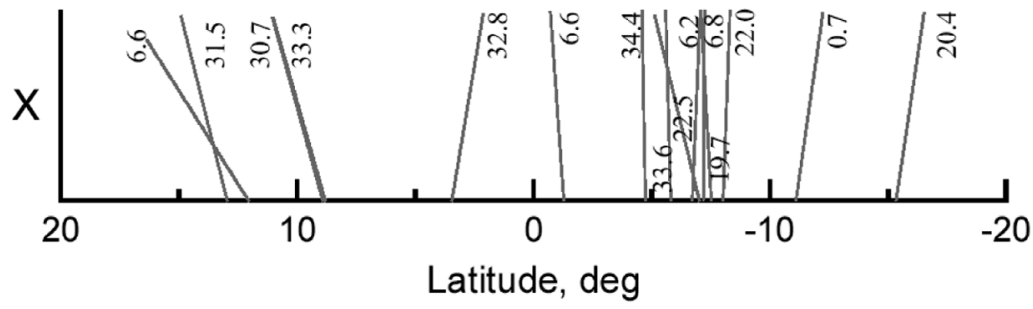

(c)

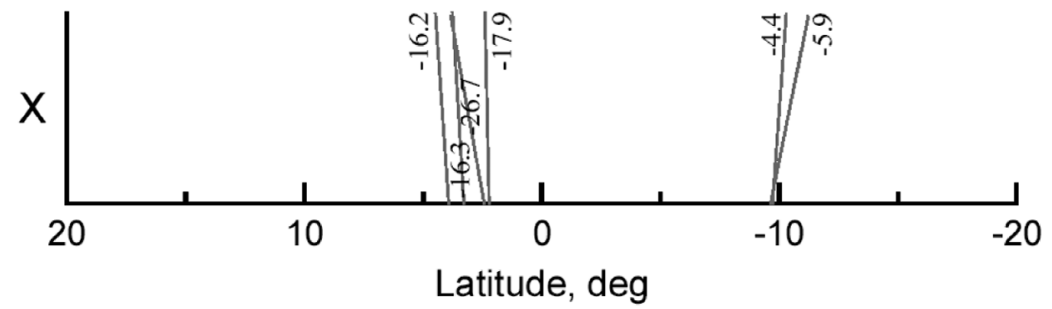

Fig. 5. Orientations of the normal to the magnetopause relative to the aGSM X-axis in the zonal plane (a) and meridional plane in the postnoon (b) and prenoon (c) sectors for strong southward IMF: $B_{z}<-5 \mathrm{nT}$ and $P_{s w}<16 \mathrm{nPa}$. The magnetopause is characterized by a strong dawn-dusk asymmetry. The numbers at lower panels indicate aGSM longitudes.

haps, this bluntness is related to a translational shift of the blunt magnetopause nose region to the postnoon sector.

The dawn-dusk asymmetry is also revealed in the orientations of the magnetopause normal in meridional plane. In the prenoon sector, the normal demonstrates a regular increase of the tilt with the module of latitude. In the post- noon sector, the normal is almost parallel to the $\mathrm{X}$-axis in the range of latitudes from $\sim-5^{\circ}$ to $\sim 10^{\circ}$. Note that in this case, the Northern hemisphere is purely covered by the data. Such orientation of the normal in the postnoon sector is an additional indication of the unusual magnetopause bluntness. Hence, we find that during intense reconnection at the 
magnetopause affected by very strong southward IMF, the magnetopause in the postnoon sector is characterized by a prominent bluntness, the extent is estimated to be from $0^{\circ}$ to $\sim 30^{\circ}$ in longitude and from $\sim-10^{\circ}$ to $\sim 10^{\circ}$ in latitude.

\section{DISCUSSION}

From the GOES observations of the magnetopause crossings, we have found two prominent distortions from the well-accepted magnetopause shape: unusual bluntness in the nose region and substantial duskward skewing of the nose region for large southward IMF. Discussing possible drivers of the asymmetry, Dmitriev et al. $(2004,2011)$ showed convincingly that asymmetries in the upstream solar wind drivers, such as IMF orientation along/orthogonal the Parker spiral, were unlikely responsible for the dawn-duskward asymmetry of the magnetopause at geosynchronous orbit. Orientation of the IMF during geosynchronous magnetopause crossings is totally different from either Parker or ortho-Parker spiral, which are usually exhibited for the undisturbed solar wind. In contrast, geosynchronous magnetopause crossings occur during magnetic storms caused by magnetic clouds whose magnetic structure is characterized by a very strong $B_{z}$ component.

Dmitriev et al. (2011) concluded that the hot plasma of storm-time partial ring current, occupying the dusk and postnoon sectors, might be a cause of the duskward translational shifting of the magnetopause. Recent advantages in remote sensing of the storm-time ring current by an energetic neutral atom (ENA) imager of the TWINS mission (Keesee et al. 2012) provided a strong support for this idea. In addition, the thermal pressure of hot plasma populating the storm-time ring current is considered as a factor which restricts and saturates the magnetic reconnection at the dayside magnetopause (Dmitriev et al. 2011).

It is reasonable to assume that the bluntness is also caused by an internal source such as region 1 FACs (Siscoe et al. 2004). On the other hand, Nakano and Iyemori (2003) showed that during magnetic storms, the magnitude of region 2 FACs was comparable with region 1 currents in the postnoon and premidnight sectors and, thus, the net contribution of the region 1 and 2 FACs was small. This finding supports the experimental result conducted by Nagai (1982) who found that no appreciable variations in the dayside geosynchronous magnetic field are associated with the Birkeland currents during geomagnetic storms.

In addition to the Birkeland currents, the ring and cross-tail currents contribute to the storm-time geomagnetic field as shown schematically in Fig. 6. During magnetic storms, the magnetopause is controlled by a pressure balance for confined geomagnetic field (Stern 1995). The magnetospheric pressure is the result of magnetic pressure $\mathrm{Pm}$ and thermal pressure Pt of hot ions in the ring current (e.g., Dmitriev et al. 2011). Hence, we can express the pressure balance in the magnetopause nose region as follows:

$k P_{s w}=\frac{H^{2}}{8 \pi}+P_{t}$

$H=\left(H_{d}+\sum H_{i}\right)+\left(f_{d} H_{d}+\sum f_{i} H_{i}\right)$

where $H_{d}$ is a geodipole magnetic field, and $H_{i}$ are magnetic field components generated by magnetospheric currents such as the ring, tail, and Birkeland currents. The magnetospheric currents are intensified in response to the IMF turning southward. The first term in the right-hand side of Eq. (3) $\left(H_{d}+\sum H_{i}\right)$ is the geomagnetic field generated by all the magnetospheric sources in the nose point. The second term depicts a contribution of shielding currents at the magnetopause which confine the geomagnetic field inside the magnetosphere. Shielding parameters $f_{d}$ and $f_{i}$ depend on the magnetopause shape and might change with solar wind conditions. For a "non-disturbed" magnetosphere $\left(H_{i}=0\right)$, Eq. (3) is converted into a classical pressure balance equation with the shielding parameter $f_{d}=2 f-1$ such that $f_{d}=$ 1(2) for the planar (spherical) magnetopause.

The shielding parameters also depend on the configuration of the magnetic field. During magnetic storms, the ring and cross-tail currents are very intense and their magnetic effect at the dayside magnetopause might become comparable with the effect of a geodipole field (Maltsev et al. 1996; Turner et al. 2000). A magnetic effect of the ring current is still a controversial topic which depends on its spatial extent. The ring current, localized in the inner drift shells $(L<6)$ increases the magnetic field at the magnetopause. Widely distributed current extending up to the dayside mag-

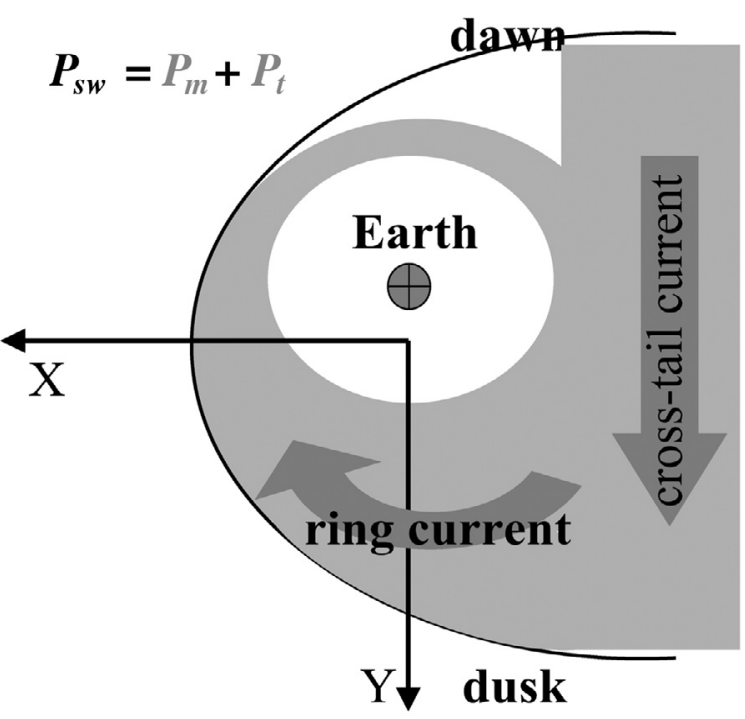

Fig. 6. A sketch of the storm-time magnetopause affected by the ring and cross-tail currents. 
netopause would produce the opposite magnetic effect. A depletion of the dayside geomagnetic field results also from a storm-time intensification of the cross-tail current and from a sunward motion of its inner edge such that the enhanced current approaches to the dayside magnetopause.

The geometry of the magnetic field produced by the storm-time cross-tail current at the magnetopause is apparently different from the dipole. The field is rather close to a linear configuration formed near the edge of a large-scale flat electric current. Such a flat current produces almost constant magnetic effect at different latitudes. Hence, the shielding parameter for the magnetic field generated by the cross-tail current at the nose magnetopause should approach $f_{d}=1$. In addition, the negative magnetic effect of the "linear" magnetic field from the cross-tail curent should be largest near the magnetic equator, where the dipole magnetic field is the weakest.

Hence, it is reasonable to propose that the unusual magnetopause bluntness results from a strong contribution of the cross-tail current to the magnetic field at the dayside magnetopause. This idea allows also an explanation of the blunt magnetopause during strong magnetospheric compression by the high solar wind pressure, which, in turn, causes a fast intensification of the cross-tail current (Borovsky et al. 1998; Tsyganenko 2000).

\section{CONCLUSIONS}

GOES magnetic data during magnetopause crossings allow finding the following distortions of the magnetopause affected by strong southward IMF:

(1) An unusual bluntness (with very large radius of curvature) extending from $0^{\circ}$ to $\sim 30^{\circ}$ in longitude and from $-10^{\circ}$ to $10^{\circ}$ in latitude;

(2) Substantial duskward skewing of the nose region;

An unusual bluntness is also revealed for high solar wind pressure and weak southward or northward IMF.

The effect of duskward translational shifting as well as reconnection saturation can be explained by a substantial contribution of the hot magnetospheric plasma from the strong asymmetrical ring current into the pressure balance at the dayside magnetopause during magnetic storms. The unusual bluntness can result from the magnetic effect of the intensified cross-tail current.

Acknowledgements This work was supported by grants NSC-99-2111-M-008-013 and NSC-100-2111-M-008-016 from the National Science Council of Taiwan and by Ministry of Education under the Aim for Top University program at National Central University of Taiwan.

\section{REFERENCES}

Borovsky, J. E., M. F. Thomsen, and R. C. Elphic 1998: The driving of the plasma sheet by the solar wind. J. Geophys. Res., 103, 17617-17639, doi: 10.1029/97JA029 86. [Link]

Borovsky, J. E., M. Hesse, J. Birn, and M. M. Kuznetsova, 2008: What determines the reconnection rate at the dayside magnetosphere? J. Geophys. Res., 113, A07210, doi: 10.1029/2007JA012645. [Link]

Dmitriev, A. V. and A. V. Suvorova, 2000: Three-dimensional artificial neural network model of the dayside magnetopause. J. Geophys. Res., 105, 18909-18918, doi: 10.1029/2000JA900008. [Link]

Dmitriev, A. V., J. K. Chao, and D. J. Wu, 2003: Comparative study of bow shock models using Wind and Geotail observations. J. Geophys. Res., 108, 1464, doi: 10.1029/2003JA010027. [Link]

Dmitriev, A. V., A. V. Suvorova, J. K. Chao, and Y. H. Yang, 2004: Dawn-dusk asymmetry of geosynchronous magnetopause crossings. J. Geophys. Res., 109, A05203, doi: 10.1029/2003JA010171. [Link]

Dmitriev, A. V., J. K. Chao, M. Thomsen, and A. Suvorova, 2005a: Geosynchronous magnetopause crossings on 29-31 October 2003. J. Geophys. Res., 110, A08209, doi: 10.1029/2004JA010582. [Link]

Dmitriev, A. V., I. S. Veselovsky, and O. S. Yakovchouk, 2005b: Inconsistency in the solar wind parameters between data sets OMNI and OMNI-2. In: Proc. of Pulkovo Solar Conference, Pulkovo, Russia, July 1520, 2005.

Dmitriev, A. V., J. K. Chao, A. Suvorova, K. Ackerson, K. Ishisaka, Y. Kasaba, H. Kojima, and H. Matsumoto, 2005c: Indirect estimation of the solar wind conditions in 29-31 October 2003. J. Geophys. Res., 110, A09S02, doi: 10.1029/2004JA010806. [Link]

Dmitriev, A. V., A. V. Suvorova, and J. K. Chao, 2011: A predictive model of geosynchronous magnetopause crossings. J. Geophys. Res., 116, A05208, doi: 10.10 29/2010JA016208. [Link]

Keesee, A. M., J. G. Elfritz, D. J. McComas, and E. E. Scime, 2012: Inner magnetosphere convection and magnetotail structure of hot ions imaged by ENA during a HSSdriven storm. J. Geophys. Res., 117, A00L06, doi: 10. 1029/2011JA017319. [Link]

Kuznetsov, S. N. and A. V. Suvorova, 1998: An empirical model of the magnetopause for broad ranges of solar wind pressure and $\mathrm{Bz}$ IMF. In: Moen, J., A. Egeland, and M. Lockwood (Eds.), Polar Cap Boundary Phenomena, 51-61, Kluwer Academic Publishers, Norwell, Mass.

Lin, R. L., X. X. Zhang, S. Q. Liu, Y. L. Wang, and J. C. Gong, 2010: A three-dimensional asymmetric magnetopause model. J. Geophys. Res., 115, A04207, doi: 10.1029/2009JA014235. [Link]

Maltsev, Y. P., A. A. Arykov, E. G. Belova, B. B. Gvozdevsky, and V. V. Safargaleev, 1996: Magnetic 
flux redistribution in the storm time magnetosphere. J. Geophys. Res., 101, 7697, doi: 10.1029/95JA03709. [Link]

Nagai, T., 1982: Local time dependence of electron flux changes during substorms derived from multi-satellite observation at synchronous orbit. J. Geophys. Res., 87, 3456-3468, doi: 10.1029/JA087iA05p03456. [Link]

Nakano, S. and T. Iyemori, 2003: Local time distribution of net field-aligned currents derived from high-altitude satellite data. J. Geophys. Res., 108, 1314, doi: 10.1029/2002JA009519. [Link]

Ober, D. M., N. C. Maynard, W. J. Burke, G. R. Wilson, and K. D. Siebert, 2006: "Shoulders" on the high-latitude magnetopause: Polar/GOES observations. J. Geophys. Res., 111, A10213, doi: 10.1029/2006JA011799. [Link]

Omidi, N., T. Phan, and D. G. Sibeck, 2009: Hybrid simulations of magnetic reconnection initiated in the magnetosheath. J. Geophys. Res., 114, A02222, doi: 10.10 29/2008JA013647. [Link]

Rufenach, C. L., Jr., R. F. Martin, and H. H. Sauer, 1989: A study of geosynchronous magnetopause crossings. $J$. Geophys. Res., 94, 15125-15134, doi: 10.1029/JA094i A11p15125. [Link]

Šafránková, J., Z. Němecek, S. Dušík, L. Přech, D. G. Sibeck, and N. N. Borodkova, 2002: The magnetopause shape and location: A comparison of the Interball and Geotail observations with models. Ann. Geophys., 20, 301-309, doi: 10.5194/angeo-20-301-2002. [Link]

Shue, J.-H., P. Song, C. T. Russell, J. K. Chao, and Y. H. Yang, 2000: Toward predicting the position of the magnetopause within geosynchronous orbit. J. Geophys. Res., 105, 2641-2656, doi: 10.1029/1999JA900467. [Link]

Shue, J.-H., P. Song, C. T. Russell, J. T. Steinberg, J. K. Chao, G. Zastenker, O. L. Vaisberg, S. Kokubun, H. J. Singer, T. R. Detman, and H. Kawano, 1998: Magnetopause location under extreme solar wind conditions. J. Geophys. Res., 103, 17691-17700, doi: 10.1029/98 JA01103. [Link]
Siscoe, G., J. Raeder, and A. J. Ridley, 2004: Transpolar potential saturation models compared. J. Geophys. Res., 109, A09203, , doi: 10.1029/2003JA010318. [Link]

Sonnerup, B. U. Ö. and B. G. Ledley, 1974: Magnetopause rotational forms. J. Geophys. Res., 79, 4309-4314, doi: 10.1029/JA079i028p04309. [Link]

Stern, D. P., 1995: Modeling the field of the global magnetopause. In: Song, P., B. U. Ö. Sonnerub, and M. F. Thomsen (Eds.), Physics of the Magnetopause, 45-51, AGU Geophysical Monograph 90.

Suvorova, A. V., A. V. Dmitriev, J. K. Chao, Y. H. Yang, and M. Thomsen, 2003: Necessary conditions for the geosynchronous magnetopause crossings. Abstract on EGS-AGU-EUG Joint Assembly, Nice, France, 7-11 April 2003.

Suvorova A. V., A. V. Dmitriev, J. K. Chao, M. Thomsen, and Y. H. Yang, 2005: Necessary conditions for geosynchronous magnetopause crossings. J. Geophys. Res., 110, A01206, doi: 10.1029/2003JA0100 79. [Link]

Tsyganenko, N. A., 2000: Solar wind control of the tail lobe magnetic field as deduced from Geotail, AMPTE/IRM, and ISEE 2 data. J. Geophys. Res., 105, 5517-5528, doi: 10.1029/1999JA000375. [Link]

Tsyganenko, N. A. and M. I. Sitnov, 2005: Modeling the dynamics of the inner magnetosphere during strong geomagnetic storms. J. Geophys. Res., 110, A03208, doi: 10.1029/2004JA010798. [Link]

Tsyganenko, N. A., H. J. Singer, and J. C. Kasper, 2003: Storm-time distortion of the inner magnetosphere: How severe can it get? J. Geophys. Res., 108, 1209, doi: 10.1029/2002JA009808. [Link]

Turner, N. E., D. N. Baker, T. I. Pulkkinen, and R. L. McPherron, 2000: Evaluation of the tail current contribution to Dst. J. Geophys. Res., 105, 5431-5439, doi: 10.1029/1999JA000248. [Link]

Yang, Y. H., J. K. Chao, A. V. Dmitriev, C. H. Lin, and D. M. Ober, 2003: Saturation of IMF $B_{z}$ influence on the position of dayside magnetopause. J. Geophys. Res., 108, 1104, doi: 10.1029/2002JA009621. [Link] 